\title{
Les populations naturelles de Stenasellus virei Dollfus (Crustacé Asellote troglobie)
}

\author{
par
}

\author{
GUY MAGNIEZ*
}

\section{GÉNÉRALITÉS}

Lorsque parût le mémoire de Racovitza sur le genre Stenasellus (1950), l'étude de l'espèce virei se basait sur le matériel provenant des récoltes effectuées dans 15 grottes ${ }^{1}$. Onze de ces stations n'avaient permis de capturer que quelques individus: 2 à Castel-Mouly (8), 2 également à l'Haiouat de Pelou (13), 4 au Solencio de Morrano (5), par exemple, malgré des visites répétées, au cours de plusieurs campagnes annuelles d'explorations. Certaines de ces visites étaient même restées infructueuses.

$\mathrm{Au}$ gouffre de Padirac (1) même, il ne fût guère possible de préciser l'importance des peuplements, par suite de la profondeur des eaux. Il ne restait donc les grottes du Mont-de-Chac: (3) et de l'Estelas (6) ${ }^{2}$ qui pouvaient permettre l'observation d'une population importante de St. virei, cantonnée dans une réserve liquide bien délimitée, de faible profondeur et accessible au chercheur sur son pourtour entier. Ces captures, qui portaient sur 198 individus à l'Estelas et 71 au Mont-de-Chac, permirent à Racovitza de formuler les remarques suivantes:

1. Absence quasi-générale d'individus de taille inférieure à $5 \mathrm{~mm}$. Tout au plus peut-on signaler, parmi les captures, quelques mâles de $4,5 \mathrm{~mm}$ et par exception, 2 femelles immatures de $4 \mathrm{~mm}$ seulement, sur le total des individus cités plus haut.

2. Tous les mâles sont adultes. La plupart atteignent $6-7 \mathrm{~mm}$. Cette dernière longueur paraît être le maximum connu pour ces stations. Leurs appendices copulateurs ont atteint leur conformation définitive dans tous les cas observés.

1 Dans le courant de 1967, j'ai établi une première liste des stations connues, tant karstiques qu'interstitielles, de Stenasellus virei. Leur nombre se montait alors à 77 et j'ai donné une description sommaire du biotope correspondant à chacune. Cette première énumération a été suivie, en 1971, d'une seconde, portant le nombre des stations à 105. Dans le présent texte, le nom des stations citées est suivie de leur numéro d'ordre chronologique, afin de permettre de les retrouver rapidement dans les deux articles descriptifs en question (voir bibliographie).

2 Dont j'ai fait mention précédemment (Magniez 1968), dans l'étude morphologique consacrée à l'espèce polytypique St. virei, car Racovitza (1950) avait redécrit en détail l'espèce d'après des individus de ces deux stations pyrénéennes, alors que Dollfus l'avait fait en premier lieu sur les individus de Padirac. La Grotte de Gourgue (15) était signalée par Racovitza comme une bonne station. Elle a depuis lors souffert de l'excès des visites, quoique d'une année à l'autre, quelques individus y soient toujours visibles.

\footnotetext{
* Laboratoire de Biologie Animale et Générale Faculté des Sciences de l'Université de Dijon, 6 Bd. Gabriel, 21000 Dijon, France
} 
3. La grande majorité des femelles sont des adultes, dont les oostégites sont déjà visibles (taille de 6 à $7 \mathrm{~mm}$ ).

4. A la grotte du Mont de Chac, environ les $2 / 5$ des femelles adultes sont munies d'un marsupium et portent des oeufs, des embryons ou des pulli. Cela indique qu'une activité de reproduction importante existe, au moins dans cette station.

Dans ces conditions, on pouvait s'étonner de l'absence, parmi ces abondantes récoltes, d'individus jeunes, de taille comprise entre 1,6 et $4 \mathrm{~mm}$ et remarquer l'exceptionnelle rareté de ceux de $4-5 \mathrm{~mm}$. Une explication était immédiate: les collectes ayant été faites à vue, seuls les plus grands individus avaient été capturés. Les plus petits devaient, soit être indiscernables sur le fond limoneux des flaques, soit vivre en permanence dissimulés profondément dans leurs terriers, comme les jeunes Niphargus. Plusieurs années d'observations, portant en particulier sur des populations cavernicoles confinées, ainsi que la récolte quasi-totale de l'une d'entre elles, permettent de préciser les raisons de l'anormale rareté des jeunes Sténaselles dans ces populations cavernicoles d'eau libre. La découverte de peuplements phréatiques et hyporhéiques, dont la composition en classes de taille est tout à fait identique à celle des Asellides épigés permet une intéressante comparaison avec ces populations des grottes. Enfin, l'évolution naturelle des élevages expérimentaux de St. virei semble en conformité avec la composition de ces populations cavernicoles confinées.

\section{LES POPULATIONS NATURELLES D’ASELLIDES ÉPIGÉS}

Les données numériques précises sur la composition des populations naturelles d'Asellides, dans les biotopes particuliers à chaque espèce, sont rares. Chacun a pu voir, à la saison convenable, le pullulement des formes communes, comme Asellus aquaticus L., dans certaines eaux stagnantes de surface et constater la présence, dans ces populations, d'une grande quantité d'individus très jeunes. Pourtant, beaucoup d'espèces, principalement les hypogées, vivent en des milieux tels que la collecte simultanée d'animaux suffisamment nombreux demeure impossible, soit par suite de l'incapacité du prospecteur à accéder au véritable biotope de l'espèce, soit à cause de la faible densité du peuplement. C'est le cas pour Proasellus spelaeus (Racovitza) ou P. albigensis (Magniez), par exemple, dont le nombre d'individus a pu permettre une diagnose et une description suffisantes, mais reste bien inférieur à ce qu'une étude de population exigerait.

Steel (1961), cherchant à préciser les étapes du cycle biologique de deux espèces épigées: Asellus aquaticus et Proasellus meridianus (Racovitza), fut amené à effectuer d'importantes ponctions mensuelles dans de riches peuplements de ces Aselles, communs dans certaines eaux douces des Iles Britanniques.

Les mensurations de tous les individus d'un prélèvement mensuel, groupés par classe de taille de $1 / 2 \mathrm{~mm}$, sont exprimées par l'auteur sous forme d'un histogramme de fréquence. 
L'un des buts de Steel fut d'abord, en comparant les divers histogrammes mensuels, de mettre en évidence l'existence d'un important maximum saisonnier de la reproduction, pour ces deux espèces dont le cycle vital se déroule approximativement sur une anrée. Bien que de tels résultats soient délicats à comparer à ce que laissent entrevoir les espèces du genre Stenasellus dont nous disposons (caractère hypothétique de la rythmicité de la reproduction, déroulement de la vie sur de nombreuses années), il convient de reprendre rapidement les enseignements de l'étude de Steel:

1. En dehors de la crise annuelle de reproduction, les échantillons d'Aselles présentent une distribution normale dont le mode se déplace de mois en mois vers les grandes tailles (de $4 \mathrm{~mm}$ en octobre à $6 \mathrm{~mm}$ en mai, pour $P$. meridianus, par exemple).

2. Les populations étudiées après l'éclosion de la grande masse de la génération annuelle montrent un considérable pourcentage d'individus juvéniles (plus de 70\% en juin, pour la même espèce). Ce taux décroît dès les mois suivants, puisque ces jeunes deviennent très vite matures (fig. $1 \mathrm{~A}$ ).

3. Lorsque deux générations se chevauchent dans le temps (en mai, par exemple, pour $A$. aquaticus), la répartition de l'échantillon est nettement bimodale (fig. $1 \mathrm{C}$ ).

\section{LES POPULATIONS HYPORHÉIQUES DE ST. VIREI VIREI ET DE ST. VIREI BOUI}

Une étude quantitative précise, telle que Steel l'a réalisée sur des épigés exigeait des échantillons de quelques centaines ou de quelques milliers d'individus. Nous avons reconnu qu'elle demeurait impossible pour la plupart des Asellotes hypogés, faute de pouvoir collecter simultanément et dans un même lieu un grand nombre d'individus. Cette difficulté se retrouve pour l'étude de St. virei. Si les stations sont désormais nombreuses, les populations à la fois denses et accessibles restent rares. Citons parmi les plus récemment découvertes la Cueva de Cullalvera, pour St. virei buchneri. Les échantillons qu'elles peuvent procurer restent donc un matériel vivant précieux, à réserver tout d'abord à l'étude du cycle biologique de l'espèce. Les prélèvements dans les milieux interstitiels ou parafluviaux, à condition que l'appareillage d'aspiration du liquide phréatique soit assez puissant, permettent pourtant de préciser quelques aspects qualitatifs de la nature des peuplements interstitiels de Stenasellus virei:

1. Sur une dizaine de prélèvements, correspondant à autant de stations de ce type, 8 n'ont procuré que quelques individus ou même un exemplaire unique du Crustacé. Les 2 autres correspondent à des ensembles de plusieurs dizaines d'individus ${ }^{1}$.

\footnotetext{
1 Une de ces stations hyporhéiques (no. 75) mise en évidence par Bou et Rouch a été étudiée récemment (Gourbault et Lescher-Moutoué 1967). Des prélèvements régulièrement échelonnés sur 3 mois ont permis d'y capturer 1301 Stenasellus virei boui, sans que le biotope paraisse épuisé. Cette importante population sera étudiée ultérieurement.
} 


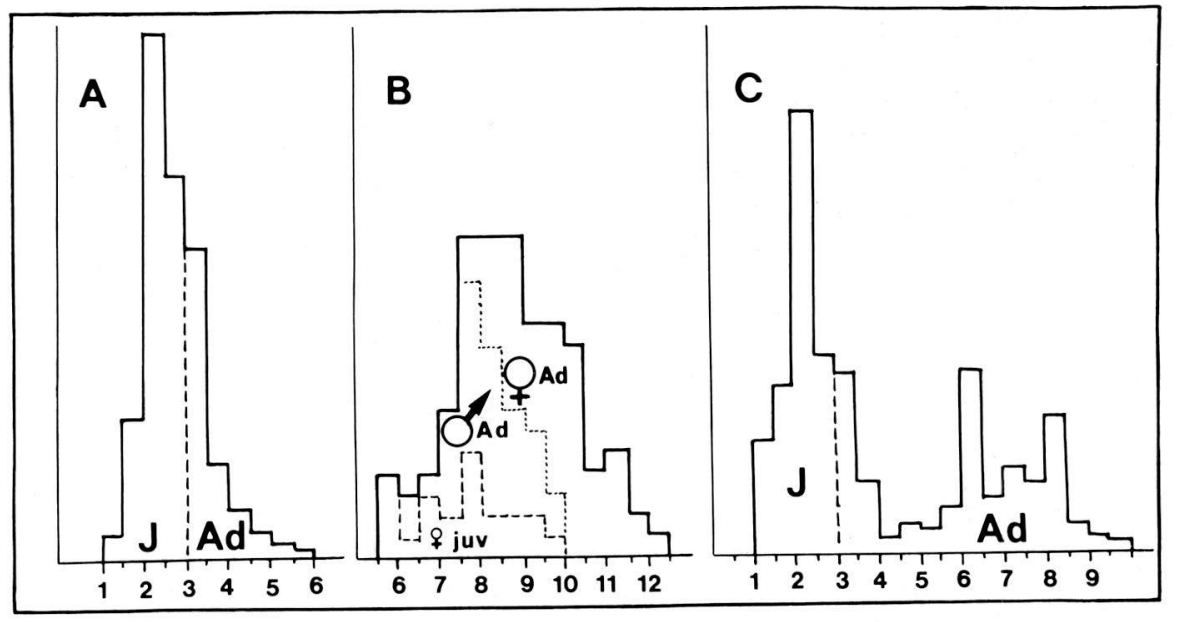

Fig. 1. A. Composition en classes de taille de $1 / 2 \mathrm{~mm}$, d'une population épigée de Proasellus meridianus (Racovitza), au mois de juin, (d'après Steel 1961, modifié). On remarque l'importance considérable du nombre des individus jeunes (70\% du total). La maturité sexuelle se produit pour une taille de $3 \mathrm{~mm}$. Toutes les catégories de taille possibles sont représentées, depuis les plus petites (pulli mesurant entre 1 et $1,5 \mathrm{~mm}$ ).

B. Composition en classes de taille de $1 / 2 \mathrm{~mm}$, d'une population cavernicole de Stenasellus virei hussoni, vivant en eau libre au siphon du Goueil-di-Her (Haute-Garonne). Toutes les catégories de taille comprises entre 1,6 et 5,5 $\mathrm{mm}$ sont absentes. Les seuls individus immatures de la population sont des femelles sans oostégites de taille déjà importante $(5,5$ à $9,6 \mathrm{~mm})$. Selon les données fournies par les élevages, une telle population ne comprendrait aucun individu âgé de moins de 5 ans.

C. Composition en classes de taille de $1 / 2 \mathrm{~mm}$, d'une population épigée d'Asellus aquaticus L., au mois de mai (d'après Steel 1961, modifié). Toutes les classes supŕieures à $5 \mathrm{~mm}$ représentent des individus nés en fin d'été de l'année précédente et qui sont destinés à disparaître prochainement (cette génération est déjà disparue de l'échantillon de $P$. meridianus considéré dans la Fig. 1 A). La génération de printemps de l'année en cours montre une composition normale où toutes les classes de taille, depuis les pulli sont représentées.

Les échelles des abscisses des 3 histogrammes représentent les longueurs des individus en millimètres. En ordonnées sont les fréquences des différentes classes.

Dans tous les lots, tous les individus ont été examinés et mesurés. Environ les $2 / 3$ sont des jeunes dont le sexe n'est pas encore reconnaissable extérieurement (les plus petits ne mesurent que $1,6 \mathrm{~mm}$ et n'ont pas encore subi leur première mue), ou des subadultes de 3 à $5 \mathrm{~mm}$, de sexe déjà discernable (les mâles n'ont pas encore terminé leur différenciation externe et les femelles n'ont point encore acquis leurs oostégites). L'autre tiers se compose d'adultes parfaits: (mâles, femelles en période 
de repos génital, mais aussi femelles ovigères ou à marsupium vide), démontrant ainsi que la reproduction s'effectue normalement dans ce biotope.

2. Dans le cas plus favorable où le prélèvement fournit simultanément un grand nombre d'individıs (nappe alluviale du Nert, du Salat), on retrouve cette même coexistence d'individus juvéniles et d'adultes, avec des proportions très comparables ( $1 / 3$ d'adultes), la taille maximale atteignant dans ce cas $7,5 \mathrm{~mm}$ pour les mâles et $9 \mathrm{~mm}$ pour les femelles.

3. Conclusion: Dans ces populations hyporhéiques de St. virei, les stades juvéniles et subadultes sont abondamment représentés et leur pourcentage est du même ordre que celui donné par Steel pour les populations épigées de Proasellus meridianus à la saison favorable. Ces populations interstitielles de St. virei peuvent donc être qualifiées de populations normales quant à leur composition en individus.

\section{LES ACCUMULATIONS D'INDIVIDUS PROVENANT DU MILIEU HYPOTHELMINORHÉIQUE}

Bien qu'il ne s'agisse pas de populations proprement dites, il y a lieu d'envisager ce cas:

1. Réservoir à Riverenert (Ariège), (48): c'est un bassin de décantation cimenté et obscur collectant les eaux d'une source qui draine un vallon humide. Les individus sont rejetés individuellement et irrégulièrement par la canalisation collectrice et s'accumulent dans le bassin où ils prospèrent. Ils peuvent alors être périodiquement prélevés et étudiés.

2. Source de Millas (60): les Sténaselles y sont recueillis par filtrage des eaux au cours de longues périodes. Les conditions sont donc les mêmes que pour le premier exemple, c'est-à-dire qu'un échantillonnage de la faune phréatique se trouve recueilli à un exutcire des eaux.

3. Station de Conłlens de Betmajou (41): Ici, une courte galerie artificielle a drainé vers elle les eaux circulantes des fissures du massif et capte donc une partie de la faune de ce milieu. Celle-ci peut être interceptée par filtrage au fond de la galerie.

4. Composition des lots recueillis: Considérons par exemple la faune rejetée par l'une des sources $(60)$, qui fournit régulièrement $S t$. virei à toute époque de l'année. Sur un total de 35 individus recueillis, il se trouve 7 femelles adultes de 5,6 à $7,2 \mathrm{~mm}, 11$ mâles de 3,5 à $6,5 \mathrm{~mm}$. 6 d'entre eux sont de très jeunes adultes, bien qu'ils possèdent déjà la conformation définitive de leurs appendices copulateurs. Il faut ajouter 8 femelles subadultes de 4,1 à $6,4 \mathrm{~mm}$ et enfin 7 jeunes de 1,6 à $1,8 \mathrm{~mm}$,âgés vraisemblablement de quelques mois seulement. Nous sommes donc en présence d'un prélèvement dont les $3 / 5$ sont constitués d'individus de petite taille et 
juvéniles. Les tailles moyennes de chacune des catégories sont remarquablement basses. Comme il est de règle, les plus grands individus sont des femelles matures $(6,5 \mathrm{~mm})$, puis viennent les mâles $(5,2 \mathrm{~mm})$ et enfin les femelles immatures $(5,1 \mathrm{~mm})$.

On peut donc constater que les lots de St. virei d'origine phréatique collectés jusqu'à présent sont de composition très semblable à celle des populations sousfluviales et parafluviales. On y rencontre simultanément des individus de toutes dimensions. Quant aux exemplaires de très grande taille, habituellement récoltés dans les populations cavernicoles, ils semblent faire défaut, soit par suite de la nature physique du milieu, soit parce qu'ils résistent mieux à un entraînement par l'eau.

\section{LES POPULATIONS DE ST. VIREI CONFINÉES EN GROTTES}

Ces populations se rapportent aux sous-espèces virei virei, pour le Gouffre de Padirac, virei hussoni pour les grottes pyrénéennes et virei buchneri pour la cavité cantabre. Elles vivent dans le biotope classique de l'espèce, biotope qui fut longtemps considéré comme le seul milieu de vie des Sténaselles, c'est-à-dire les flaques, les lacs souterrains, les nappes permanentes à fond limoneux dans lequel ces Crustacés peuvent fouir et établir leurs terriers caractéristiques. Les stations de ce type sont nombreuses (quelques 50 actuellement!) et pourtant celles qui abritent une colonie populeuse, c'est-à-dire un nombre suffisant de Sténaselles groupés sont rares $^{1}$. Citons les grottes du Mont-de-Chac et de l'Estelas (dont les peuplements n'ont donc pas périclité depuis l'époque des explorations de Jeannel et de Racovitza, de 1906 et 1912 et depuis les très importants prélèvements de Stenasellus pratiqués par Husson en septembre 1955), celles du Bédat (10) et de Cullalvera (34).

Le Gouffre du Sauvajou (54) et le Goueil-di-Her (65) contiennent également d'imposantes populations de Sténaselles, dont l'observation ne peut avoir lieu que dans des conditions exceptionnelles, par suite de la topographie de la première cavité et du régime des eaux de la seconde. Les contrôles effectués au cours de plusieurs années dans 4 des grottes citées, ainsi que l'analyse des prélèvements provenant de toutes permettront de préciser la portée des observations faites et la signification de ces populations.

\section{Composition de ces prélèvements}

1a. Gouffre du Sauvajou: plusieurs prélèvements, effectués par Bou en 1964 et 1966 portent sur une centaine d'individus. La totalité sont des adultes de 7 à $10 \mathrm{~mm}$, sauf 8 femelles sans oostégites, qui sont des subadultes de 6 à $7 \mathrm{~mm}$.

1 La liste des stations établie dans le courant de 1967 rend compte sommairement des particularités de chacune. Beaucoup de grottes baptisées "stations à St. virei", n'ont donné que quelques individus, après des prospections minutieuses et répétées. C'est insuffisant pour définir la structure des "populations" qui les habitent. 
1b. Grotte du Bédat: Sur environ 140 individus capturés, la plupart sont des adultes mâles et femelles. Il se trouve toutefois dans ce lot 30 femelles subadultes de 6 à 7,5 mm. Par exception, l'une d'elles n'atteint que $5 \mathrm{~mm}$.

1c. Cueva de Cullalvera: dans cette magnifique station de St. virei buchneri, nous avons pu prélever un lot de 130 individus. 8 d'entre eux seulement étaient des subadultes, de sexe femelle, dont une seule de $4,7 \mathrm{~mm}$ et les autres de 6 à $7,5 \mathrm{~mm}$.

1d. Gouffre de Padirac: 42 individus de St. virei virei ont été pêchés en 1963, au moyen de balances. Sur ce lot, on ne trouve qu'un immature femelle de taille déjà notable.

1e. Grotte de l'Esttelas: l'ensemble des prélèvements effectués pendant de nombreuses années représente un effectif de 750 mâles et femelles adultes, auxquels il faut ajouter 60 femelles subadultes de moins de $7 \mathrm{~mm}$. Dans ce nombre des immatures, il n'est possible d'en citer que 6 de taille comprise entre 4 et $5,5 \mathrm{~mm}$. J'ai pratiqué à plusieurs reprises et en des points différents du lac des filtrages d'importantes quantités de limon meuble provenant du fond, avec des filets à mailles suffisamment fines pour retenir les pulli. Ils n'ont rapporté qu'un seul individu de $2 \mathrm{~mm}$, de sexe encore indiscernable. Il ne semble donc pas que la masse de limon abrite des concentrations importantes d'individus juvéniles. On peut en déduire que, si les jeunes existent effectivement au sein du limon, il n'a pas été possible de les y mettre en évidence avec la densité que l'on pouvait supposer.

1f. Grotte du Mont de Chac: elle présente les conditions les meilleures pour l'observation de la population de St. virei hussoni qui l'habite. Elle est d'accès facile et le gour à fond linoneux qui abrite les Sténaselles est de faible surface: quelques $\mathrm{m}^{2}$. Il est peu profond, bien isolé des circuits aquifères souterrains. C'est donc un milieu limité, presque clos, où la population locale du Crustacé se perpétue avec une grande régularité depuis 1906 , date de la première observation.

Malgré la multiplicité des prélèvements et l'emploi de filets à mailles fines, un seul individu juvénile de $2,5 \mathrm{~mm}$ environ y a été capturé ${ }^{1}$, contre 660 de taille importante, dont une centaine de femelles sans oostégites de 5 à $7 \mathrm{~mm}$. Le fond du gour étant formé, sous une pellicule ameublie par les Crustacés, de quelques $\mathrm{cm}$ de limon cohérent, recouvrant les dépôts calcitiques, l'enlèvement ménagé de ce revêtement ${ }^{2}$, dans une partie exondée en période de basses eaux, a permis d'y trouver 3 jeunes individus de 4 , 4 , et $4,5 \mathrm{~mm}$. L'un d'eux était un jeune mâle impubère ${ }^{3}$.

\footnotetext{
${ }^{1}$ Comme au lac de l'Estelas, les jeunes individus sont donc particulièrement rares dans la couche superficielle meuble du limon.

2 De quelques $\mathrm{dm}^{2}$

${ }^{3}$ Les jeunes individus sont donc présents dans la masse de dépôt cohérent, mais avec une densité modeste, qui ne dépasse pas celle des adultes en surface.
} 
1g. Goueil-di-Her: la capture fortuite, pendant l'été de 1967, d'une importante population de Sténaselles provenant du siphon de cette grotte permet d'arriver à des constatations identiques, mais apporte d'intéressantes précisions. 108 individus ont été capturés en eau libre. 35 sont des mâles de 6 à 9,9 mm (moyenne 8,04 mm), 23 sont des femelles immatures de 5,8 à $9,6 \mathrm{~mm}$ (moyenne $7,54 \mathrm{~mm}$ ) et 50 sont des femelles adultes de 7,8 à 12,1 mm (moyenne 9,7 mm), (fig. $1 \mathrm{~B}$ ).

Or, dans le même temps, Rouch capturait dans le milieu interstitiel de cette grotte, dont le grand siphon contient d'importantes masses de graviers noyés, un lot de 75 Sténaselles. 40 sont des mâles immatures et matures de 2,9 à 5,4 mm (moyenne $3,9 \mathrm{~mm}$ ). Par exception, l'un d'eux atteignait $7 \mathrm{~mm}$. 31 sont des femelles immatures de 2,9 à 5,1 mm (moyenne 3,8 mm). Par exception, 2 d'entre-elles mesuraient 6,3 et $8,7 \mathrm{~mm}$. Enfin, 4 femelles de 4,3 à $4,5 \mathrm{~mm}$ possédaient déjà des oostégites, par suite d'une puberté exceptionnellement précoce.

\section{Les conséquences de ces observations}

Bien que l'exposé ci-dessus n'en fasse que peu mention, la recherche des individus immatures et des stades juvéniles de $S t$. virei dans toutes les cavités abritant des populations de l'espèce a été un souci constant au cours de plusieurs années de prospection ${ }^{1}$. En effet, il aurait été très utile de disposer d'emblée de jeunes exemplaires en nombre suffisant pour entreprendre des élevages expérimentaux du Crustacé ${ }^{2}$. Or, le résultat fut pratiquement négatif, aux quelques petits individus cités près. Il existe donc une discordance totale entre la composition des captures en milieu interstitiel et celles que fournissent les biotopes cavernicoles classiques en eau libre.

Dans les populations des grottes étudiées, les individus juvéniles, de taille inférieure à $4 \mathrm{~mm}$, ne sont pratiquement pas représentés. Les seuls immatures présents sont des femelles subadultes de taille déjà considérable ( $5,6 \mathrm{~mm}$ ou plus).

Récemment, j'ai pu constater que St. virei possédait un cycle vital très dilaté par rapport à celui des Asellides épigés. La maturité sexuelle demanderait quelques 5 années avant de se manifester. Ensuite, la vie adulte pourrait se dérouler sur une durée au moins équivalente, ce qui indiquerait que les membres d'une population

\footnotetext{
${ }^{1}$ C'est pourquoi des tentatives répétées de capture de ceux-ci par filtrage du limon meuble qui est au fond des flaques et par des prélèvements de la couche de dépôt cohérent, argileux ou limoneux qui se trouve en dessous, furent effectuées.

2 L'étude de la durée des mues et des intermues juvéniles, de leur nombre, de la différenciation sexuelle externe, de la croissance et même de la reproduction en aurait été beaucoup facilitée et accélérée. Au contraire, les populations cavernicoles contiennent un fort pourcentage "d'adultes âgés", en particulier de femelles de grande taille, ayant déjà élévé une portée de jeunes. Ces femelles passent ensuite par une période d'intermues de repos sexuel extrêmement longue (des années! ) au cours de laquelle s'accroît lentement une nouvelle génération d'ovocytes. Il s'agit donc d'un matériel particulièrement inerte et défavorable pour réaliser des élevages de l'espèce. Les individus des biotopes hyporhéiques seront certainement beaucoup plus intéressants pour constituer des élevages, mais la découverte de l'espèce dans ce milieu est encore trop récente pour que les captures aient été utilisées systématiquement dans ce but.
} 
cavernicole pourraient vivre 10 ans ou plus. Dans ces conditions, la composition de ces populations de grottes apparaît comme tout à fait anormale. D'une part, aucun facteur saisonnier ne peut-être invoqué pour expliquer la rareté généralisée des immatures, d'autant que les prélèvements furent faits à toutes époques de l'année et, d'autre part, les jeunes qui vivent enfouis dans le limon ne s'y trouvent qu'en nombre insuffisant par rapport à la prolificité que semble conserver l'espèce dans certaines de ces stations. Les individus des classes de taille juvéniles ne vivraient normalement pas au contact des adultes et des subadultes de taille déjà importante. Il existerait une véritable ségrégation, en fonction de la taille des individus dans ce type de population.

Les observations faites au Goueil-di-Her suggèrent que, côte à côte, dans une

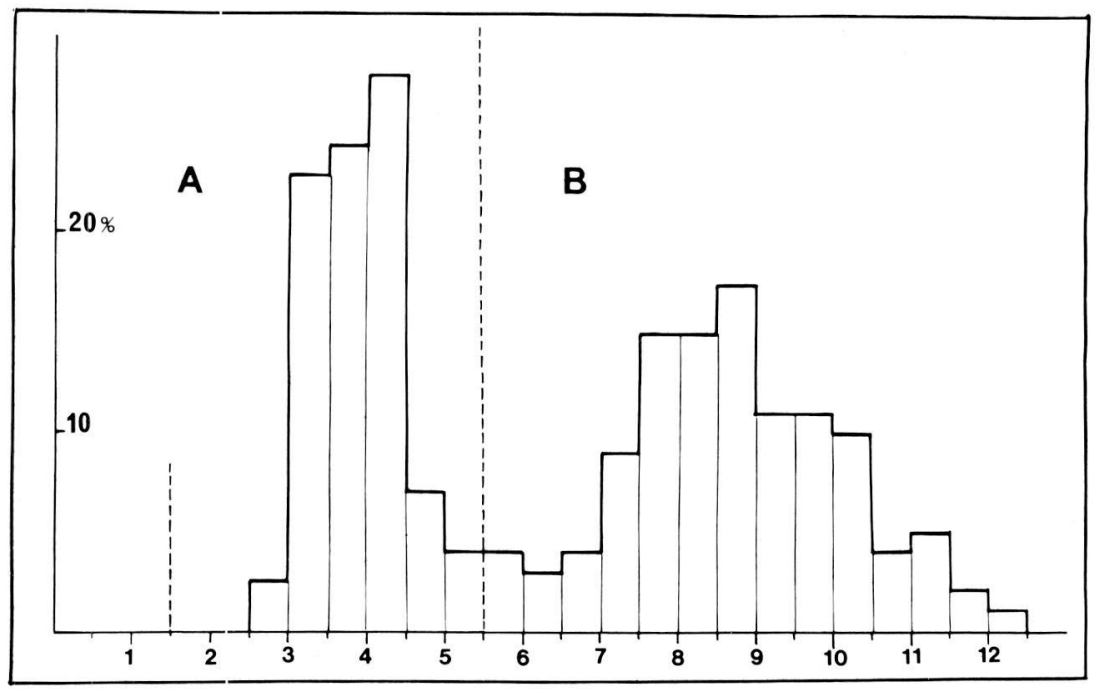

Fig. 2. Composition $€$ n classes de taille de $1 / 2 \mathrm{~mm}$; des prélèvements globaux de Stenasellus virei hussoni, effectués au siphon du Goueil-di-Her (Haute-Garonne).

A. Lot d'individus provenant du millieu interstitiel (sables et graviers du siphon). Prédominance des tailles comprises entre 3 et 4,5 $\mathrm{mm}$ (stades juvéniles et quelques adultes exceptionnellement précoces). Absence des pulli et très jeunes individus (1,6 à $2,5 \mathrm{~mm}$ ), sans doute par suite de l'imperfection des méthodes de prospection.

B. La portion de l'histogramme située à droite de la ligne pointillée verticale correspond à la figure $1 \mathrm{~B}$, ramenée à la même échelle que $2 \mathrm{~A}$. Elle représente la population des eaux libres du siphon. Les deux lots sont donc étroitement complementaires: il s'agit d'une seule population au sein de laquelle se produit une ségrégation en fonction de la taille des individus.

Le déficit des classes comprises entre 5 et $7 \mathrm{~mm}$ semble uniquement dû à des raisons pratiques (impossibilité des prélèvements dans certaines zones et à certaines profondeurs). 
même cavité, peuvent cohabiter une population d'individus de grande taille, qui sont installés en eau libre, sur le fond limoneux d'un vaste lac et, dans le milieu interstitiel des sables et graviers voisins, une autre population, de structure toute différente, dans laquelle les stades juvéniles vivent, soit seuls, soit avec un très faible nombre d'adultes dont la taille est encore modeste.

Les deux populations sont étroitement complémentaires, du point de vue de leur composition en classes de taille (fig. 2).

La signification de la ségrégation pratiquée par les jeunes Stenasellus demande à être précisée. Le cas de la grotte du Mont-de-Chac est favorable à cette recherche car la vasque qui abrite les Sténaselles constitue un biotope de dimensions restreintes, étroitement confiné et l'abri offert aux jeunes par les anfractuosités des concrétions et les dépôts limoneux est peu important et très accessible.

\section{Reproduction et cohabitation des générations de $S t$. virei en milieu confiné}

Lorsque dans une même cavité, les milieux aquatiques libre et interstitiel se présentent côte à côte avec un grand développement, la ségrégation mise en évidence ne rencontre aucune difficulté pour s'effectuer. Or, le cas est parfois différent. Ainsi, la grotte du Mont-de-Chac (et celle de l'Estelas) n'offre guère cette possibilité, par suite de l'isolement de la vasque où vit le Crustacé. Par analogie avec les formes épigées et compte tenu de la longueur de la phase juvénile des Sténaselles, la population du Mont-de-Chac devrait comprendre quelques 70\% d'immatures, vivant dans les interstices inaccessibles aux adultes, dans les fines anfractuosités de la muraille calcitique du gour, par exemple. Essayons de suivre ces jeunes individus depuis leur origine:

\section{3a. L'activité reproductrice au Mont-de-Chac}

Les importants prélèvements de faune effectués dans cette grotte contiennent un lot de femelles à poche incubatrice encore occupée par les embryons ou par les jeunes attendant leur libération prochaine. La composition de ces portées a été étudiée. Il apparaît, d'une part, qu'on peut compter sur une descendance de 26 jeunes Stenasellus, en moyenne, par portée. D'autre part, la station est réputée (Racovitza 1950) pour l'importance du nombre des femelles gestantes qu'il est possible d'y observer simultanément. Compte tenu de ce nombre, il semble vraisemblable que, dans cette flaque isolée de quelques $\mathrm{m}^{2}$ de surface seulement, dans les meilleures conditions, mais qui se réduit régulièrement, à la fin de l'été, à moins de $1 \mathrm{~m}^{2}$, avec une profondeur résiduelle de quelques $\mathrm{cm}$ seulement, ce sont quelques 800 à 1000 pulli qui doivent naître chaque année. Cette valeur minimale ne tient compte que des femelles gestantes observées. Elle est certainement beaucoup plus élevée en réalité. Si l'on se réfère aux données de Steel, la station du Mont-de-Chac devrait héberger des milliers de Sténaselles immatures de 1,6 à $5 \mathrm{~mm}$ et par conséquent 2000 à 3000 adultes, ce qui est manifestement invraisemblable. 


\section{3b. Estimation de la population adulte de St. virei dans le gour du Mont-de-Chac}

Sans qu'il soit question de détruire cette irremplaçable station, pour en dénombrer exactement la population, un raisonnement simple va en donner une valeur approximative. En effet, dans une population étroitement limitée en nombre par les nécessités vitales de chaque individu et dont chacun d'entre eux vit de nombreuses années, toute pontion d'adultes, provoquée artificiellement, doit nécessairement se répercuter sur la structure même de la population.

3b1. De 1906 à 1960, les prélèvements d'adultes furent modérés (Jeannel 1906, Jeannel et Racovilza 1912), sauf en 1955, lorsque Husson préleva à deux reprises, des lots très impcrtants de Sténaselles (plus de 150 individus), pour réaliser des élevages de l'espèce, tant au Laboratoire souterrain de Moulis qu'au Laboratoire de Biologie Animale de l'Université de Sarrebrück.

Dans les lots de Biospeologica, il existait beaucoup de femelles âgées, de forte taille (jusqu'à $11 \mathrm{~mm}$, d'après Racovitza 1950) ${ }^{1}$. Le pourcentage des femelles immatures était de $7 \%$ environ dans ces prélèvements. Une période de 5 années pleines sépare les captures de 1955 (Husson) des premières que j’ai pu réaliser. Au cours de celle-ci, la population du Mont-de-Chac a pu se reconstituer.

3b2. En 1960 et 1961, des prélèvements très importants et répétés de Sténaselles eurent lieu. Ils comportaient, à chaque fois, la collecte systématique de tous les individus visibles sur une importante fraction de la superficie du gour. 175 exemplaires, dont 98 femelles les composent. Le pourcentage des femelles immatures, qui était de $6 \%$ pour le premier prélèvement s'est élevé à $23 \%$ dans le dernier.

3b3. Dans le courant de 1963, un contrôle fut effectué. La population était peu dense en grands ndividus, néammoins 25 femelles adultes purent encore être retirées de la station.

3b4. A partir de l'été de 1964, deux ponctions très rigoureuses eurent lieu. Elles fournirent quelques 180 individus. Dans le lot des femelles, environ $70 \%$ cette fois étaient des immatures. De plus, la taille des mâles était généralement plus faible que précédemment. Il y avait manifestement, parmi cet échantillon très complet, une grande majorité d'adultes dont le passage à cet état était récent.

3b5. En 1966, les prélèvements ont été réduits. Environ $30 \%$ des femelles étaient encore des immatures. Les femelles adultes présentaient une taille encore faible (7-7,5 mm), mais la plupart étaient déjà ovigères, ce qui montre que l'activité

1 La présence d'individus de très forte taille $(11,12 \mathrm{~mm})$, dans des populations cavernicoles de St. virei, apparaît de plus en plus comme une caractéristique des populations "vierges" de Sténaselles, c'est-à-dire: de celles dont les prospections humaines n'ont point encore troublé la structure, plutôt que comme un caractère racial typique. A la grotte du Mont-de-Chac, il existait en 1906 des individus de $11 \mathrm{~mm}$; ce n'est plus vrai actuellement. 
reproductrice n'avait rien perdu de son intensité. Au cours de l'été 1967, une dernière ponction de 100 Crustacés a montré qu'alors, $27 \%$ des femelles étaient encore des immatures et que la reproduction était très active.

3b6. Conclusion: dans cette station étroitement confinée, des ponctions répétées, au cours de plusieurs années successives ont eu pour résultat un véritable remaniement de la structure de la population. Le prélèvement systématique, étalé sur 3 années, de quelques 120 femelles adultes a eu comme corollaire la raréfaction considérable de celles-ci dans la vasque (une trentaine de captures seulement), puis leur remplacement, en eau libre à la surface du gour, par des immatures, qui ont dû quitter leur refuge à cette occasion, pour venir "combler les places vides".

Une évaluation tout à fait approximative laisse prévoir que le gour n'abrite que quelques centaines d'adultes ${ }^{1}$. Comme cette population, dans l'évaluation la plus défavorable, engendre au moins 800 jeunes chaque année (certainement beaucoup plus), il faut admettre que la plus grande part d'entre eux (peut-être 90\%) disparaissent avant d'atteindre une taille notable ${ }^{2}$. Cette disparition doit même survernir très tôt après leur libération par la mère.

\section{3c. Les confirmations apportées par les élevages expérimentaux}

Les adultes réunis dans des récipients d'élevage peuvent s'y maintir durant des années. Des cas de reproduction déjà nombreux ont été constatés dans ces conditions. Les femelles gestantes mènent régulièrement à terme leurs portées, comme dans le milieu naturel. Pourtant, il n'a jamais été possible d'obtenir le maintien en vie et la croissance des Stenasellus nouveaux-nés, au sein de ces populations d'adultes en milieu confiné: tous les jeunes disparaissent rapidement de l'élevage.

Lorsque chaque femelle ovigère est isolée dans un récipient particulier, les pulli, quand ils sont libérés du marsupium, se répartissent dans l'espace limité qui est dévolu à l'ensemble. Leur nombre décroît très vite. Pour conserver efficacement des portées entières de jeunes St. virei dès qu'ils quittent la poche incubatrice, il est indispensable de les séparer immédiatement de tout individu de grande taille, y compris de la femelle-mère.

En effet, contrairement aux Asellides, les Sténaselles, surtout les femelles, manifestent des tendances carnassières très marquées. Ils sont capables de capturer et de dévorer des proies vivantes à leur échelle et j'ai acquis la certitude que les jeunes de leur propre espèce sont victimes de la voracité des adultes ou des individus plus grands qu'eux, surtout en milieu confiné. Cela se produit dans les récipients

1 Le nombre de 400, par exemple, pourrait être une valeur acceptable.

2 Sinon, il faudrait supposer que le placage argileux de la vasque du Mont-de-Chac recèle quelques 5000 Sténaselles immatures de 1,6 à $5 \mathrm{~mm}$. Ce dépôt n'atteint que quelques $\mathrm{cm}$ d'épaisseur sur $2-3 \mathrm{~m}^{2}$ de superficie. Les prélèvements et les filtrages effectués montrent qu'en réalité, les jeunes individus, s'ils sont présents dans les terriers qu'ils creusent dans ce matériau cohérent jusqu'au contact avec la calcite, ne le sont qu'avec une densité faible ( 1 par $\mathrm{dm}^{2}$, par exemple). Leur nombre total est sans doute comparable à celui des grands immatures et des adultes vivant en eau libre dans la vasque. 
d'élevage où ce confinement résulte de la nécessité de limiter les dimensions du matériel utilisé, ou dans les stations qui, comme celle du Mont de Chac, abritent une population dense, dans un espace tout à fait limité ${ }^{1}$. Dans un cas comme dans l'autre, en effet, la fréquence des rencontres entre individus est très grande et les apports nutritifs limités.

\section{3d. Conséquence}

La concurrence vitale en milieu cavernicole confiné: même si les Cyclopides, les Vers ou les Protozoaires y abondent, il est certain que la faune macroscopique aquatique du biotope à St. virei hussoni est très peu variée. Les exemples du gour du Mont-de-Chac ou du lac de l'Estelas en sont la preuve ${ }^{2}$. Précisons, en particulier, que les Amphipodes y font totalement défaut ${ }^{3}$. Il ne semble pas que la population de Sténaselles y soit soumise à l'action de prédateurs, quelle que soit leur taille. Compte tenue de leurs tendances carnassières et de leur comportement en élevage, il n'est pas absurde de penser que, dans un milieu étroitement confiné et limité, comme le montrent les deux exemples cités, la seule concurrence vitale sérieuse à laquelle soit soumise l'espèce soit une concurrence intraspécifique. Elle serait exercée par les plus grands individus sur les plus petits ou sur ceux qui se trouvent en état d'infériorité momentanée (en cas de mue), comme cela se produit en élevage.

Durant les périodes de stabilité, ce seraient le nombre et la densité des adultes qui limiteraient la pullulation des jeunes. Ceux-ci doivent donc être littéralement décimés dès leur libération du marsupium. Un équilibre spontané existerait entre le reliquat des individus juvéniles, vivant surtout dans des anfractuosités inaccessibles aux plus grands ou en permanence dans des galeries à leur taille ${ }^{4}$, et la population d'adultes et de grands subadultes installés en eau libre. Le remplacement naturel des

\footnotetext{
1 Toutes les espèces d'Asellotes ne réagissent pas ainsi. J'ai réalisé des élevages de Proasellus coxalis (Dollfus) au laboratoire souterrain de Moulis. Dans l'obscurité complète et à température constante, pourvu que la nourriture (feuilles macérées d'Orme) soit abondante et distribuée régulièrement, il se produit une véritable pullulation d'individus de cette espèce, même dans un récipient de dimensions réduites. Toutefois, dans ces conditions, les individus nés dans l'aquarium n'atteignent jamais une taille comparable à celle de leurs parents provenant d'une station épigée. Le régime no:mal de ces Aselles est strictement végétarien. En effet, en élevage, ils ne dévorent les cadavres de leurs congénères ou les mues qu'en cas de manque total de nourriture végétale dans le récipient d'expérience.
}

${ }^{2}$ Il ne semble pas que l'Oligochète Phreoryctidae Pelodrilus leruthi Hrabe, 1958, que l'on rencontre fréquemment associé à St. virei dans ses stations cavernicoles (Husson 1957), cause le moindre tort au Crustacé, à quelque stade que ce soit.

${ }^{3} \mathrm{Au}$ siphon du Goueil-di-Her, la population de St. virei hussoni est accompagnée de larves diverses d'Insectes, de quelques Niphargus sp. de petite taille et d'une population de Proasellus racovitzai Henry et Magniez. Comme la surface disponible et le volume de l'eau sont normalement imposants, la densité des individus de chaque espèce reste infime.

${ }^{4}$ Cette seconde éveritualité rendant leur survie plus aléatoire, puisque les galeries des grands individus risquent de recouper les leurs. 


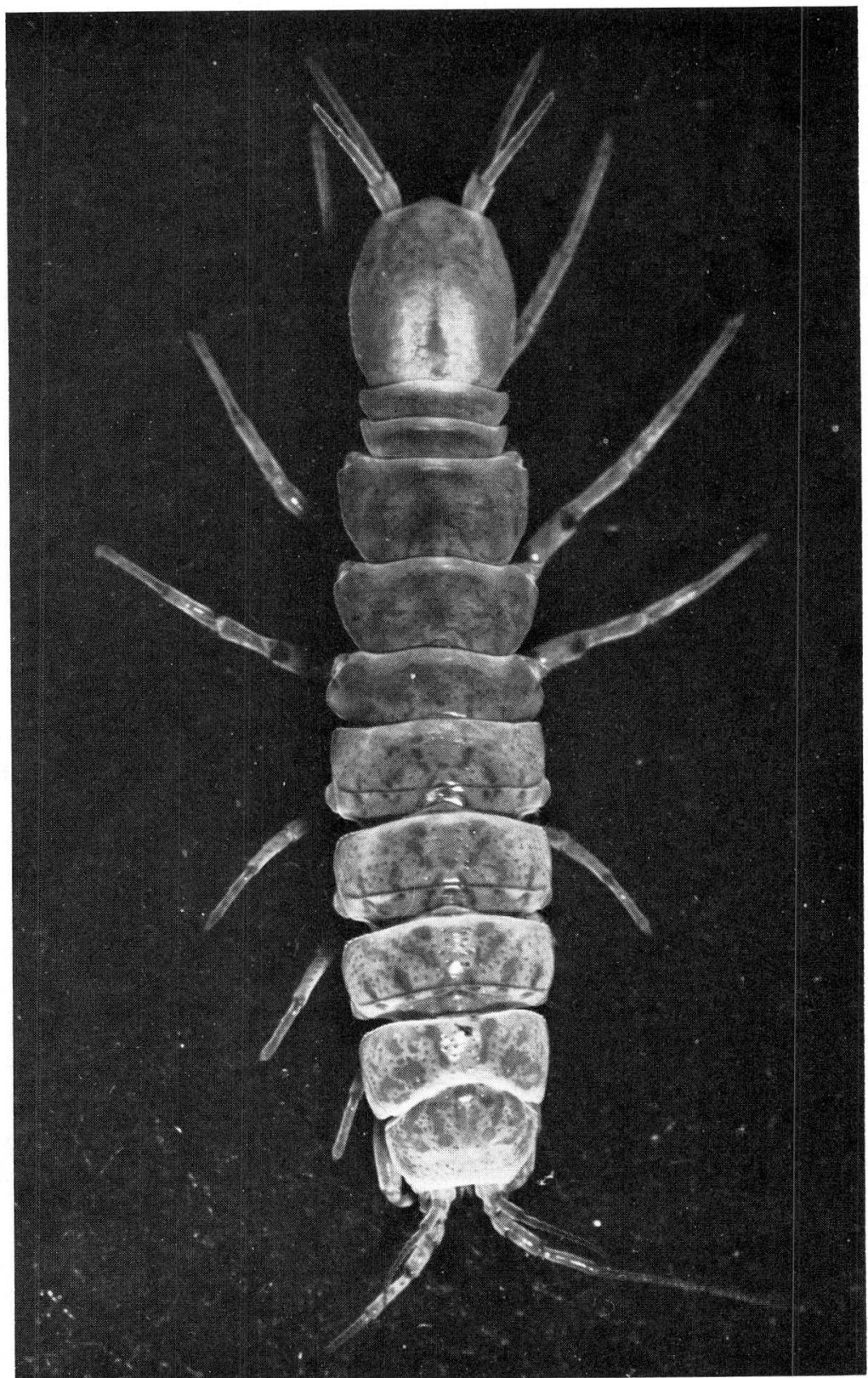

Fig. 3. Stenasellus virei hussoni: grande $\$$ adulte de $10 \mathrm{~mm}$ environ, provenant de la population vivant en eau libre dans le siphon de la grotte du Goueil-di-Her (Haute-Garonne), population étudiée dans le présent article. 
individus séniles se trouverait ainsi assuré et il suffirait pour cela d'une faible proportion des générations successives de jeunes.

Les ponctions expérimentales d'adultes troubleraient profondément le processus en multipliant les chances de survie dans les classes d'âge juvénile ${ }^{1}$. Cette question pourra sans doute être précisée ultérieurement.

Les cas où la population cavernicole de Stenasellus vit dans un biotope étroitement confiné, comme au Mont-de-Chac ou à l'Estelas est un cas limite, bien qu'il existe réellement dans les réseaux fossiles. Souvent, les masses d'eau des grottes où vivent les Crustacés communiquent plus ou moins facilement, à la saison humide par exemple, entre elles ou avec des réseaux aquifères actifs, ou encore sont liées à des graviers et sables noyés sous-jacents. La survie des jeunes individus dans de telles conditions reste très difficile à étudier, mais on peut supposer que la possibilité des migrations la rend moins aléatoire. L'étude des populations interstitielle et d'eau libre capturées au Goueil-di-Her semblerait illustrer ce cas.

\section{3e. Le cas de St. virei en milieu interstitiel}

La présence en abondance de Sténaselles (St. virei virei, virei boui et virei angelieri) dans certains milieux hypothelminorhéiques et hyporhéiques est une acquisition trop récente pour faire l'objet d'un développement. Pourtant, à titre de comparaison, il faut rapeller deux points essentiels:

- D'une part, en milieu interstitiel, les Sténaselles doivent trouver un espace à 3 dimensions qui n'existe pratiquement pas pour de nombreuses stations en grottes. Les individus de toutes tailles doivent s'installer dans les interstices à leur convenance, en fonction des caractéristiques granulométriques tout à fait locales. Ceci permet d'envisager la ségrégation et, donc, la survie des jeunes individus que l'on rencontre effectivement en nombre dans les prélèvements interstitiels.

- D'autre part, ce milieu abrite une faune extrêmement riche et variée, tant au point de vue des groupes représentés que de la densité du peuplement (Angelier 1953, Delamare-Deboutteville 1960, Bou et Rouch 1967). Les populations de Stenasellus s'y trouvent donc en concurrence avec des formes variées (Cyclopides, Harpacticides, Microparasellides, Amphipodes Niphargus sp., Salentinella sp., Ingolfiella $s p$., pour donner quelques exemples de Crustacés). Certaines de ces espèces sont suceptibles de servir de proies aux Sténaselles, mais ceux-ci peuvent aussi être les victimes des formes plus robustes. Ce sont donc des rapports hétérospécifiques qui peuvent s'exprimer ici. Il semble que Stenasellus virei rencontre en milieu interstitiel des conditions de vie fondementalement différentes de celles qu'il subit en milieu cavernicole strict.

\footnotetext{
1 Naturellement, elles ne mettent pas en danger l'existence de la station si elles ne sont pas répétées systématiquement au cours d'une durée supérieure à la période de vie adulte normale des Sténaselles. Il serait fort intéressant de pouvoir éliminer totalement, dans une station déterminée, suffisament isolée, les individus de taille supérieure à une certaine valeur, pour étudier ensuite le retour progressif à l'équilibre de la population qui l'habite.
} 


\section{CONCLUSIONS}

Les récentes prospections et les observations sur le terrain ont permis d'améliorer nos connaissances sur les peuplements de Stenasellus virei dans leurs milieux de vie naturels.

Les seules populations directement observables sont celles qui vivent dans des collections d'eaux permanentes de la zone de percolation des massifs karstiques. Dans ce type de biotope, les véritables populations, c'est-à-dire les peuplements durables qui se renouvellent essentiellement par reproduction in situ, sont rares. La plupart des 'stations' de cette catégorie ne correspondent, en fait, qu'à de petites accumulations d'individus ayant migré des systèmes aquifères du massif vers les collections d'eau de la grotte où ils sont plus ou moins étroitement prisonniers.

Les populations karstiques des zones amphibie et noyée, les populations interstitielles des nappes alluviales et éluviales sont les plus importantes. Toutefois, elles ne sont pas directement accessibles. On ne peut les connaître que par des échantillons recueillis par divers artifices: piégeage, filtrage continu des émergences, sondages tubés.

Ces échantillons provenant de biotopes où l'eau se trouve à l'état divisé (milieu perméable en petit, par exemple), présentent toujours une composition normale en classes de taille, c'est-à-dire avec une large prédominance des stades jeunes sur les adultes. Cette composition est conforme à celle qui est connue pour les Asellides épigés.

Dans ces biotopes, l'espèce fait partie d'une biocénose complexe de formes hypogées très variées. Elle doit pouvoir y trouver des proies convenables (Copépodes, Microparasellides, par exemple), mais elle doit y être soumise à l'action des formes prédatrices plus robustes (Amphipodes, Planaires). Les Sténaselles s'y trouvent donc soumis aux conditions d'une concurrence vitale multispécifique normale.

Les populations karstiques (St.virei buchneri et St.virei hussoni) des collections d'eau plus ou moins isolées dans la zone dénoyée des massifs présentent une composition inhabituelle en classes de taille: elles sont formées d'une grande majorité d'adultes auxquels s'ajoute un faible pourcentage de subadultes de taille déjà importante. Les jeunes y sont anormalement rares, aussi efficace que soit la technique de capture. Lorsque aucun prélèvement n'y a été encore fait, ces populations se comportent comme des accumulations stabilisées d'adultes atteignant la plus grande taille possible ( $\%$ de $12 \mathrm{~mm}$ ) et leur âge naturel maximal. Les ponctions répétées d'individus dans ces peuplements sont responsables d'une diminution rapide de la taille moyenne et provoquent un accroissement du pourcentage des immatures présents dans le groupe, c'est-à-dire un relèvement du taux de remplacement dans la population. Cette observation montre qu'il s'agit de populations dont le nombre d'individus est étroitement limité et apporte une preuve de leur confinement plus ou moins parfait dans la collection d'eau.

Dans ces colonies isolées, la prolificité de l'espèce peut demeurer bien supérieure à ce que nécessite le simple maintien de l'effectif de la population confinée. Une part très importante de chaque génération de jeunes Sténaselles disparaît très tôt, principalement par cannibalisme. En effet, les adultes ne semblent pas soumis à une 
concurrence vitale hétérospécifique normale dans ce cas, par suite de la rareté ou même de l'absence d'autres espèces prédatrices de leur taille. La concurrence vitale à laquelle est soumis St.virei hussoni à la Grotte du Mont-de-Chac, par exemple, paraît-être de type intraspécifique. Elle cause un amenuisement rapide du nombre des jeunes, dès leur libération par la mère.

$\mathrm{Au}$ niveau des zones amphibie et noyée des massifs karstiques, le milieu offre des abris très variés aux jeunes Sténaselles (dans l'interstitiel des rivières souterraines, par exemple) et l'on peut observer une ségrégation entre classes de taille qui assure la survie normale des plus petites. Les véritables peuplements karstiques sont donc ceux des zones inférieures du massif et ceux des réseaux de fissures où l'eau se trouve à l'état divisé. Les colonies visibles en grottes ne sont que des peuplements secondaires, écologiquement accessoires.

\section{RESUME}

Parmi les nombreuses stations cavernicoles ou interstitielles de Stenasellus virei, quelques unes ont été étudiées en détail pendant plusieurs années. Certaines montrent des populations relativement denses. Les peuplements endémiques des eaux permanentes des réseaux fossiles semblent avoir une composition anormale, car ils comportent surtout des individus de grande taille. Ils diffèrent des peuplements intersitiels dont la composition en classes de taille paraît normale.

Il se pourrait que ces différences de structure des populations soient liées, d'une part aux différences de nature physique des millieux constités par les eaux libres des grottes et les eaux. interstitielles et, d'autre part, aux différences de nature et de densité des associations d'espèces qui peuvent subsister respectivement dans chacun de ces deux types de milieu hypogé liquide.

\section{SUMMARY}

Many cavernicolous and phreatic localities are known for the species Stenasellus virei. Some of these, which harbor a rather abundant population have been studied for several years.

The endemic pcpulations from permanent waters of some fossil karstic systems seem to have an abnormal composition. They include especially large individuals (juvenile stages being rare). They differ from the phreatic populations, which exhibit a normal distribution in size groups with a normal percentage of juveniles.

These differences in the structure of populations may result from physical differences between the habitat in free waters of caves and in phreatic water, and from differences between the associations of species that these two types of hypogean habitat may support. 


\section{BIBLIOGRAPHIE}

ANGELIER, E. 1953. Recherches écologiques et biogéographiques sur la faune des sables submergés. Arch.Zool.exp.gen. 90, 2, 37-161.

BOU, C. et ROUCH, R. 1967. Un nouveau champ de recherches sur la faune aquatique souterraine. C.R. Acad. Sci., 265, 369-370.

DELAMARE-DEBOUTEVILLE, C. 1969. Biologie des eaux souterraines littorales et contlinentales. Hermann, Paris, 1-740.

DELAY, B. 1969. Sur le peuplement des circulations d'eau de la zone de percolation temporaire des massifs karstiques. C.R. Acad. Sci., 268, 1917-1920.

GOURBAULT, N. et LESCHER-MOUTOUE, F. 1968. Etude de la faune hypogée peuplant le sous-écoulement du Nert. Ann. Spéléol., 23, 4, 735-742.

HENRY, J.P. et MAGNIEZ, G. 1970. Contribution à la systématique des Asellides (Crustacea Isopoda). Ann. Spéléol., 25, 2, 335-367.

HUSSON, R. 1957. A propos de la biologie du Crustacé troglobie aquitique Stenasellus virei Dollfus. Ann. Univ. Saraviensis, Sc., 6, 74, 8259-69.

LESCHER-MOUTOUE, F. 1968. La faune d'une nappe éluviale de déversement: étude préliminaire des Cyclopides hypogés. Ann. Spéléol., 23, 4, 743-751.

MAGNIEZ, G. 1967/1971. Les stations de Stenasellus virei Dollfus (Crustacé Isopode troglobie). Sous le Plancher, Dijon, VI/3 et VI/4, 36-48 et 49-58, 1967; VII/1, 1-11; VII/2, 21-28; VII/3, 38-40, 1968 et X/1, 13-24, 1971.

MAGNIEZ, G. 1968. L'espèce polytypique Stenasellus virei Dollfus 1897 (Crustacé Isopode hypogé). Ann. Spéléol., 23, 2, 363-407.

ORGHIDAN, T. 1959. Ein neuer Lebensraum des unterrirdischen Wassers: der Hyporheische Biotop. Arch. Hydrobiol., 55, 392-414.

RACOVITZA, E.G. 1950. Asellides première série: Stenasellus. Arch. Zool. exp. gen., 87, 1-94.

ROUCH, R. 1968. Sur les eaux souterraines continentales et l'importance de leur peuplement. Ann. Spéléol., 23, 4, 693-703.

STEEL, E.A. 1961. Some observations on the life-history of Asellus aquaticus (L.) and Asellus meridianus Racovitza (Crustacea Isopoda). Proc. Zool. Soc. London, 137, 1, 71-87.

VANDEL, A. 1964. Biospéologie. La biologie des animaux cavernicoles. Gauthier Villars, Paris, 1-619. 